\title{
Mean, individual pulses and spectrum of Geminga radio emission
}

\author{
V.M. Malofeev and O.I. Malov \\ Pushchino Radio Astronomy Observatory, Lebedev Physical Institute, \\ 142292, Pushchino, Moskow reg., Russia
}

\begin{abstract}
The measurements of profiles at 102, 87, 59 and $40 \mathrm{MHz}$ are presented. Geminga shows unique character of radio emission: the most steep spectrum, the large changes of pulse widths, phases of pulse time of arrival and the presence of giant pulses.
\end{abstract}

After the presentation of our first Geminga observations (Malofeev \& Malov, 1997) we continued the investigations of this pulsar and present new interesting data briefly. The measurements were carried out using the Large Phased Array (Pushchino) at $102 \mathrm{MHz}$. New observation confirmed the temporal changes of the form, width and the pulse phase. A few examples of integrated profiles are presented at Fig.1. The integration of 30000 periods is not enough to obtain the stable mean profile (two last profiles at Fig.1). The interpulse separated at $\sim 180^{\circ}$ from the main pulse is displayed for the last series of observations. The distribution of pulsewidths and pulse phases (Fig.2) show abnormal very broad profile at $102 \mathrm{MHz}$.

Our observations at the another radio telescope (cross-type) permit to find the radio emission of Geminga at $40,60,87 \mathrm{MHz}$ with similar pecularities mentioned above. To understand the unstable form and width of the integrated profiles we carried out the observations of individual pulses or the groups of pulses. These observations have shown positive results at all frequencies during a few percentage of the observing time (Fig.3). The most impressionable effect is the existence of the giant pulses during $\sim 0.1 \%$ of observing time (Fig.4), when the flux density can reach $\sim 10 \mathrm{Jy}$. It is worth noting that giant pulses can exist during all period, the examples are presented at Fig.4a (the second giant pulse) and Fig. 3a (the first pulse).

Geminga shows the most steep spectrum among all pulsars. Spectral index is $\geq 5$. Spectrum shows low-frequency turnover with a maximum about $60 \mathrm{MHz}$ (Malofeev - these proceedings).

Acknowledgments. This work was partly supported by RFBR (Pr No 97-02-17372) and INTAS (grant No 96-0154).

\section{References}

Malofeev V.M., Malov O.I. 1997, Nature, 389, 697. 

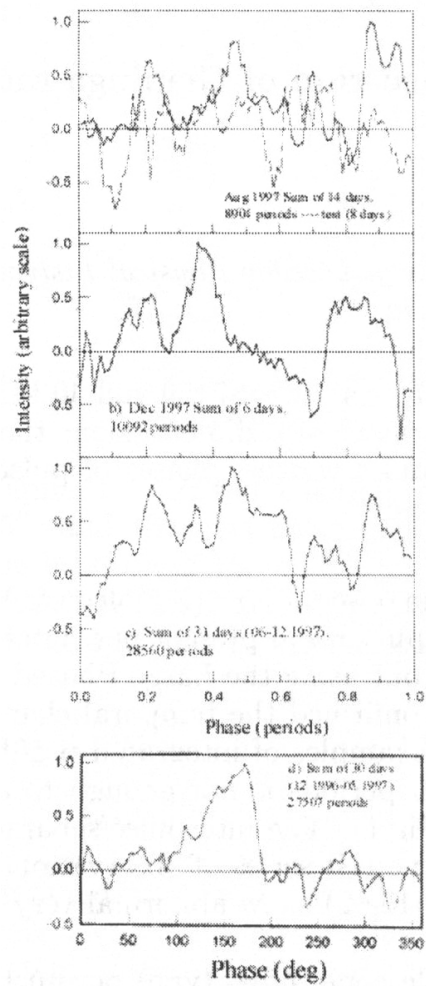

Fig. 1. Mem palse profites in new series (a, b. o). The sums of inegrated protiles (c, d).

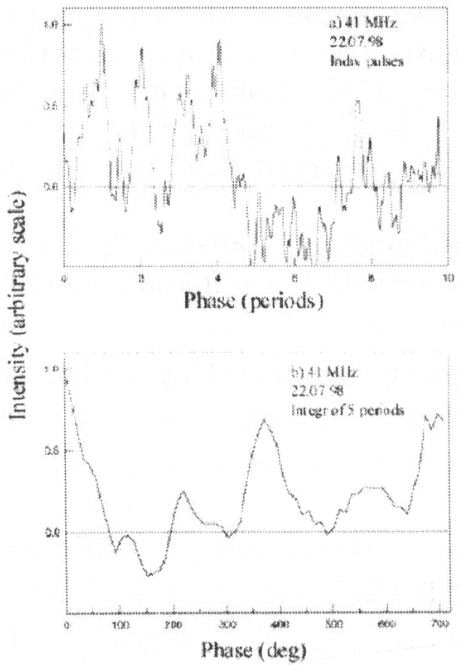

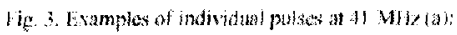
integrated protiles at $41 \mathrm{klh}$ (h) Arows dewn and ap are shom phases of the onats pulac and the interpulse.
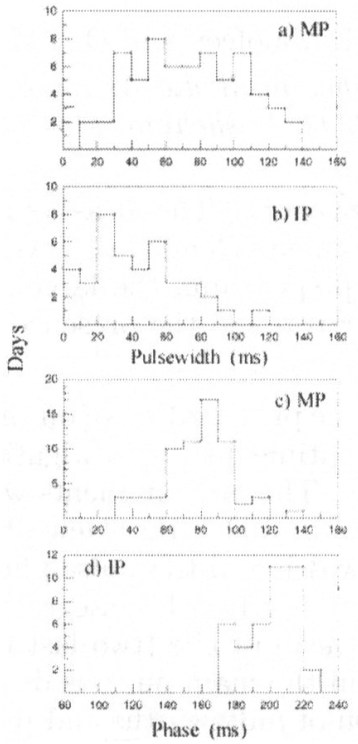

Fig. 2. Dixtributions of the pulseridth (a. by and phases of pulse tine of arrival (c. d) for main (MP) and interpulse (IP).

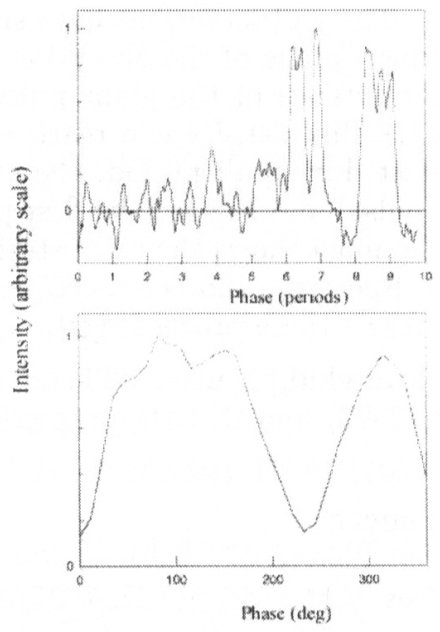

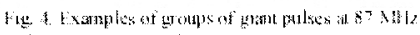

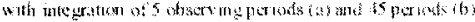

